\title{
Management of Loco-Regional Nasopharyngeal Carcinoma-The Role of Induction Chemotherapy-A Mini-Review
}

Frank E Mott ${ }^{1 *}$, Ruth L. Sacks ${ }^{2}$

'Thoracic and Head/Neck Medical Oncology, University of Texas MD Anderson Cancer Center, Texas, USA

${ }^{2}$ University of Texas MD Anderson Cancer Center, Texas, USA

\section{Article Info}

\section{Article Notes}

Received: July 20, 2018

Accepted: August 23, 2018

\section{${ }^{*}$ Correspondence:}

Dr. Frank E Mott, MD FACP, Associate Professor, Thoracic and Head/Neck Medical Oncology, University of Texas MD Anderson Cancer Center, 1515 Holcombe Blvd, Unit 432, Houston, TX 77030, USA; Telephone No: 713-792-6363; Email: fmott@mdanderson.org.

(c) 2018 Mott FE. This article is distributed under the terms of the Creative Commons Attribution 4.0 International License.

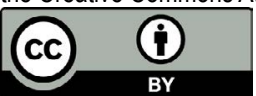

\section{Keywords:}

Nasopharyngeal Carcinoma

Induction Chemotherapy

Chemoradiation

Outcomes

\section{Abstract}

Standard treatment of locally advanced nasopharyngeal carcinoma is patterned after the Intergroup 0099 trial with concurrent cisplatin with radiotherapy, followed by consolidation chemotherapy. This remains the guideline recommended standard. With the wider use of intensity modulated radiotherapy and the incorporation of taxanes in the chemotherapy regimen, many oncologists are increasingly using an induction approach with chemotherapy followed by concurrent chemoradiotherapy. Induction chemotherapy is not considered a standard approach despite studies showing efficacy and tolerability. In this mini-review, we summarize the data from trials and reviews of induction therapy and argue for its recognition as a viable standard option for patients with locally advanced nasopharyngeal carcinoma.

Nasopharyngeal carcinoma (NPC) accounts for $0.6 \%$ of all cancers worldwide and 87,000 new cases annually. It is a male predominant disease (2.3:1) with the highest rates among populations in SouthEastern Asia, Micronesia/Polynesia, and Northern Africa ${ }^{1}$. In the United States, the incidence is $0.5-2$ per 100,000 compared to 25 per 100,000 in southern China $^{2}$. Arising from the nasopharynx epithelium, NPC morphologically demonstrates lymphoid cells intermixed with transformed epithelial cells. Generally considered squamous in origin, NPC is categorized into three different pathologic subtypes per World Health Organization (WHO) criteria based on the degree of differentiation ${ }^{3}$. Undifferentiated, non-keratinizing (WHO Type III) NPC is the most common subtype accounting for two-thirds of patients in the United States and $95 \%$ of patients in China ${ }^{4}$.

In endemic populations, there are several contributory risk factors leading to the development of NPC including Epstein Barr virus (EBV) infection, environmental factors, and genetic predisposition ${ }^{3}$. The presence of EBV DNA and EBV gene expression in precursor lesions and tumor cells suggests that EBV activation is part of the pathogenesis of nasopharyngeal carcinoma ${ }^{3,5}$. This has spurred further research to evaluate and assess the benefit of monitoring circulating EBV DNA for screening, treatment response, and recurrence $\mathrm{e}^{6-8}$.

Magnetic Resonance Imaging (MRI) is an essential component of the assessment of NPC. It helps distinguish NPC from other tumor types in addition to illustrating tumor extension and stage. In $6-10 \%$ of patients, the nasopharyngeal mucosa appears normal on endoscopy; therefore, MRI is vital in detecting subclinical tumors9. The apparent diffusion coefficient (ADC) on MRI serves as a non- 
invasive prognostic parameter to help distinguish between poor, moderate, and well-differentiated tumors. ADC correlates with other prognostic factors such as tumor type, grade, volume, and nodal status; for example, a lower ADC value reflects a lower increased cellular density with subsequent restricted diffusion on MRI, which correlates with poorly differentiated tumors, larger tumor volume, and metastatic cervical lymph nodes ${ }^{10}$.

The staging of NPC is based on the Tumor-NodeMetastasis (TNM) system of the American Joint Committee on Cancer (AJCC) Staging ${ }^{11}$. Stage I NPC demonstrates 90 percent five-year overall survival (OS) with radiation alone $^{12}$. Stages II-IV are typically treated with combined chemotherapy and radiation. According to the Meta-analysis of Chemotherapy in Nasopharynx Carcinoma (MAC-NPC), which analyzed 19 randomized trials including 4806 patients with non-metastatic nasopharyngeal carcinoma, the addition of chemotherapy to radiation improved overall survival (HR 0.79, 95\% CI 0.73-0.86, p<0.0001), reduced loco-regional failure, and reduced distant failure ${ }^{13}$. Two phase III randomized trials illustrated a survival benefit for concurrent chemoradiation (CRT) compared to radiation therapy (RT) alone in Stage II or greater NPC ${ }^{14,15}$.

The National Comprehensive Cancer Network (NCCN) 2018 guidelines recommend concurrent chemoradiation (cisplatin) followed by adjuvant chemotherapy (cisplatin/5-FU) for locoregionally advanced disease based on the Intergroup study 0099 published in $1998^{16}$. This phase III trial randomized patients with stage III and IV NPC to concurrent chemoradiation followed by adjuvant chemotherapy versus radiation alone. Threeyear progression free survival (PFS) (69\% vs. 24\%, $\mathrm{P}<0.001$ ) and three-year overall survival (OS) (76\% vs. $46 \%, \mathrm{P}<0.001$ ) both favored the combined modality $\mathrm{arm}^{17}$. However, only half of the patients in the combined modality arm completed all three cycles of adjuvant chemotherapy due to treatment toxicity. In Singapore, a study based on the structure of the Intergroup study 0099 confirmed that the addition of chemotherapy significantly improves 2-year distant metastasis-free survival (DMFS), disease free survival (DFS), and OS rates ${ }^{18}$. Similar to the findings of the MAC-NPC meta-analysis, it is not surprising that both of these studies demonstrate superiority of combined chemoradiation over definitive radiation alone. However, these studies failed to address the question of whether concurrent chemoradiation plus adjuvant chemotherapy is more beneficial than concurrent chemoradiation alone for locally advanced NPC. A Phase III trial from China demonstrated no improvement in the five-year failure-free rate with the addition of adjuvant chemotherapy compared to observation alone following concurrent chemoradiation ${ }^{19,20}$. Future studies need to better assess if there is a subset of patients that would derive benefit from adjuvant chemotherapy despite the treatment toxicities. Two trials have implemented EBV DNA as a marker of residual disease status to guide management in locoregionally advanced disease. The Hong Kong Nasopharyngeal Cancer Study Group (HKNPCSG) 0502 trial randomized patients with residual detectable EBV DNA, following concurrent CRT or RT, to 6 cycles of adjuvant chemotherapy or observation alone. The study demonstrated no significant difference in the 5-year relapse free survival (49.3\% vs. 54.7\%; HR for relapse or death $1.09,95 \%$ CI $0.63-1.89 ; \mathrm{p}=0.75)$ or overall survival $(64 \%$ vs $67.8 \%$; HR 1.09 , 95\% CI 0.56-2.11; $\mathrm{p}=0.79$ ) between the two groups. However, the study highlighted barriers to completion of adjuvant chemotherapy including treatment related toxicity and patient refusal. Only $65.4 \%$ of patients assigned to receive adjuvant chemotherapy completed 4 cycles of treatment. Nevertheless, survival outcomes did not drastically differ for patients that received more than 3 cycles of adjuvant chemotherapy based on sensitivity analysis of survival outcomes according to compliance to adjuvant chemotherapy ${ }^{21}$. The ongoing trial NRGHN001 (NCT02135042) is assessing the role of adjuvant chemotherapy following CRT based on EBV DNA plasma levels. High risk patients with persistently detectable EBV DNA are being randomized to adjuvant chemotherapy and patients with no detectable EBV DNA are being randomized to either standard adjuvant chemotherapy or observation ${ }^{22}$. Hopefully, the results of this study will provide insight into EBV DNA as a potential risk stratification tool in the setting of adjuvant chemotherapy.

The role of induction chemotherapy, while increasingly common, remains controversial. Among expert groups such as the European Society of Medical Oncology (ESMO), the National Cancer Institute (NCI), and NCCN, there is no unifying consensus on the utilization of induction chemotherapy in the management of NPC. Earlier phase III trials of induction chemotherapy followed by RT alone did not show any difference in overall survival (OS) compared to definitive RT alone ${ }^{23-25}$. However, these trials were in the pre-Intensity Modulated Radiotherapy (IMRT) era and used older anthracycline containing regimens. The MAC-NPC meta-analysis included six induction trials that demonstrated improved progression-free survival (PFS) but not OS. ${ }^{13}$ Based on the available data, it remains debated whether or not more modern taxane-containing induction regimens along with the use of concurrent chemotherapy with RT would provide a greater benefit. Uncontrolled trials using the docetaxel-platinum-5 Fluorouracil (TPF) induction regimen followed by IMRT with concurrent cisplatin have demonstrated 3-year PFS and OS rates of approximately $75-79 \%$ and $86-87 \%$ respectively ${ }^{26-28}$. In one randomized phase II trial comparing induction cisplatin-docetaxel followed by concurrent cisplatin-RT vs concurrent chemoradiotherapy alone, both PFS and OS were 
improved with the induction regimen; 3-year PFS 88.2\% vs $59.5 \%$ and 3 -year OS $94.1 \%$ vs $67.7 \%{ }^{29}$. Randomized trials comparing induction chemotherapy followed by concurrent chemoradiotherapy versus definitive chemoradiotherapy alone have been few in number and with conflicting results. Two randomized phase II studies evaluating induction therapy with triplet chemotherapy regimens failed to show benefit in either PFS or $\mathrm{OS}^{30,31}$. A phase II trial presented at the American Society of Clinical Oncology (ASCO) 2017 meeting studied induction cisplatin-gemcitabine followed by 70 Gy RT with concurrent high dose cisplatin every three weeks $^{32}$. Five-year overall survival was 70\%; local regional control was 69\%; and relapse free survival was 52\%. One hundred and seven $(35.7 \%)$ of patients failed treatment, including 48 (45\%) with distant metastases. More recently, a randomized, phase III Chinese trial with induction TPF followed by RT with concurrent high dose cisplatin every three weeks showed a 3-year failure free survival of $80 \%$ vs $72 \%(\mathrm{p}=0.034)$ favoring the induction $\mathrm{arm}^{33}$. The GORTEC 2006-02 trial also randomized 83 patients between induction TPF followed by concomitant cisplatin-RT vs concomitant therapy alone and showed a 3-year PFS and OS of $73.9 \%$ and $86.3 \%$ vs $57.2 \%$ and $68.9 \%$, respectively, statistically favoring the induction arm, $(p=0.042$ for PFS and 0.05 for OS). Ninety-five percent of patients completed all three cycles of the induction chemotherapy and grade III toxicities between the two arms were not different ${ }^{34}$. We recently reported a retrospective study of our experience with induction chemotherapy followed by chemoradiotherapy or radiotherapy alone over a ten-year period (2005-2015) in 95 patients $^{35}$. Induction regimens varied but platinum and taxane containing regimens were the most common (76\% of patients). IMRT was standard. Three-year PFS and DMFS were $77.3 \%$ and $78 \%$, respectively, comparable to data from other induction trials. Toxicities were manageable with $87 \%$ of patients completing the full induction chemotherapy course and 97\% going on to CRT/RT.

The data supporting additional chemotherapy outside of concurrent chemoradiation remains varied and there are limited comparisons of concurrent chemoradiation with induction versus concurrent chemoradiation with adjuvant chemotherapy. The MAC-NPC meta-analysis attempted to compare induction and adjuvant therapy but differences in trial designs, chemotherapy regimens, radiation methods, and follow up did not allow for an accurate assessment ${ }^{11}$. The only well-established fact is the benefit of multimodality therapy, involving both chemotherapy and radiation, over single modality therapy, involving radiation or chemotherapy alone. The guideline recommendations for the past twenty years have supported concurrent chemo-radiation followed by adjuvant/consolidation chemotherapy as first line treatment based on the Intergroup 0099 trial, in spite of a number of trials showing that induction chemotherapy followed by concurrent chemo-radiation can be as effective and with an acceptable toxicity profile. Induction regimens containing a taxane agent have consistently out-performed regimens without a taxane and should be considered as a potential first line option. This has become a de-facto standard of care in many centers and community practices. It is recommended that the guidelines be updated to reflect this practice. Many practitioners are currently implementing induction chemotherapy in patients presenting with large bulky tumors, extensive nodal disease, or those unable to receive full dose radiation due to close proximity of critical structures as well as those encountering delays in radiotherapy due to dental extractions. In the future, to better address the sequence and administration of therapy, clinical trials may need to randomize patients receiving concurrent chemoradiation into 3 arms: induction therapy, adjuvant therapy, or observation. This strategy will more accurately shed light on the ideal treatment approach to improve survival outcomes. Currently, the HKNPCSG 0501 trial is investigating this issue by comparing induction-concurrent chemotherapy vs. adjuvant-concurrent chemotherapy.

\section{References}

1. Ferlay J, Soerjomataram I, Dikshit R, et al. Cancer incidence and mortality worldwide: sources, methods and major patterns in GLOBOCAN 2012. Int J Cancer. 2015 Mar; 136(5): E359-86. Epub 2014 Oct 9

2. Chang ET, Adami HO. The enigmatic epidemiology of nasopharyngeal carcinoma.Cancer Epidemiol Biomarkers Prev. 2006; 15(10): 1765.

3. Wei WI, Sham JS. Nasopharyngeal carcinoma. Lancet. 2005; 365(9476): 2041.

4. Chua M, Wee J, Hui E, et al. Nasopharyngeal Carcinoma. Lancet. 2016; 387: 1012-24.

5. Raghupathy R, Hui EP, Chan AT. Epstein-Barr virus as a paradigm in nasopharyngeal cancer: from lab to clinic. Am Soc Clin Oncol Educ Book. 2014.

6. Chan AT, Ma BB, Lo YM, et al. Phase II study of neoadjuvant carboplatin and paclitaxel followed by radiotherapy and concurrent cisplatin in patients with locoregionally advanced nasopharyngeal carcinoma: therapeutic monitoring with plasma Epstein-Barr virus DNA. J Clin Oncol. 2004; 22(15): 3053.

7. Chan KC, Lo YM, et al. Circulating EBV DNA as a tumor marker for nasopharyngeal carcinoma.Semin Cancer Biol. 2002; 12(6): 489.

8. Chan KCA, Woo JKS, King A, et al. Analysis of Plasma Epstein-Barr Virus DNA to Screen for Nasopharyngeal Cancer. N Engl J Med. 2017; 377(6): 513

9. Abdel K, Abdel RA, King A. MRI and CT of nasopharyngeal carcinoma. AJR Am J Roentgenol. 2012; 198: 11-8.

10. Abdel RA, Kamal E. Nasopharyngeal carcinoma: correlation of apparent diffusion coefficient value with prognostic parameters. Radiol Med. 2013; 118: 534-9.

11. American Joint Committee on Cancer (AJCC) Staging Manual, $7^{\text {th }}$ edition, 2010.

12. Lee AW, Szw WM, Au JS, et al. Treatment results for nasopharyngeal carcinoma in the modern era: the Hong Kong experience. Int J Radiat Oncol Biol Phys. 2005; 61(4): 1107.

13. Blanchard P, Lee A, Marguet S, et al. Chemotherapy and radiotherapy 
in nasopharyngeal carcinoma: an update of the MAC-NPC metaanalysis. MAC-NPC Collaborative Group. Lancet Oncol. 2015; 16(6): 645. Epub 2015 May 6.

14. Chan AT, Leung SF, Ngan RK, et al. Overall survival after concurrent cisplatin-radiotherapy compared with radiotherapy alone in locoregionally advanced nasopharyngeal carcinoma. J Natl Cancer Inst. 2005 Apr 6; 97(7): 536-9.

15. Lin JC, Jan JS, Hsu CY, et al. Phase III study of concurrent chemoradiotherapy versus radiotherapy alone for advanced nasopharyngeal carcinoma: positive effect on overall and progressionfree survival. J Clin Oncol. 2003 Feb 15; 21(4): 631-7.

16. NCCN Guidelines, Cancer of the Nasopharynx, version 2018; National Comprehensive Cancer Network, 2018.

17. Al-Sarraf M, LeBlanc M, Giri PG, et al. Chemoradiotherapy versus radiotherapy in patients with advanced nasopharyngeal cancer: phase III randomized Intergroup study 0099. J Clin Oncol. 1998 Apr; 16(4): 1310-7.

18. Wee J, Tan EH, Tai BC, et al. Randomized trial of radiotherapy versus concurrent chemoradiotherapy followed by adjuvant chemotherapy in patients with American Joint Committee on Cancer/International Union against cancer stage III and IV nasopharyngeal cancer of the endemic variety.J Clin Oncol. 2005 Sep 20; 23(27): 6730-8.

19. Chen L, Hu CS, Chen XZ, et al. Concurrent chemoradiotherapy plus adjuvant chemotherapy versus concurrent chemoradiotherapy alone in patients with locoregionally advanced nasopharyngeal carcinoma: a phase 3 multicentre randomised controlled trial. Lancet Oncol. 2012; 13(2): 163. Epub 2011 Dec 7.

20. Chen L, Hu CS, Chen XZ, et al. Adjuvant chemotherapy in patients with locoregionally advanced nasopharyngeal carcinoma: Long-term results of a phase 3 multicentre randomised controlled trial. Eur J Cancer. 2017; 75: 150. Epub 2017 Feb 22.

21. Chan TC, Hui EP, Ngan R, et al. Analysis of Plasma Ebstein-Barr Virus DNA in Nasopharyngeal Cancer After Chemoradiation to Identify High-Rish Patients for Adjuvant Chemotherapy: A randomized control trial. Journal of Clinical Oncology. 2018 Jul 10.

22. Individualized Treatment in Treating Patients With Stage II-IVB Nasopharyngeal Cancer Based on EBV DNA. NRG Oncology. https://clinicaltrials.gov/ct2/show/ NCT02135042?term $=$ NCT02135042\&rank=1

23. Langendijk JA, Leemans CR, Butler J, et al. The additional value of chemotherapy to radiotherapy in locally advanced nasopharyngeal carcinoma: a meta-analysis of the published literature. J Clin Oncol. 2004; 22: 4604-12.

24. International Nasopharynx Cancer Study Group. 1; VUMCA I Trial. Preliminary results of a randomized trial comparing neoadjuvant chemotherapy (cisplatin, epirubicin, bleomycin) plus radiotherapy vs. radiotherapy alone in stage IV ( $>$ or $=\mathrm{N} 2, \mathrm{M} 0)$ undifferentiated nasopharyngeal carcinoma: a positive effect on progression-free survival. Int J Radiat Oncol Biol Phys. 1996; 35: 463-9.

25. Chua DT, Sham JS, Choy D, et al. Preliminary report of the Asianoceanian clinical oncology association randomized trial comparing cisplatin and epirubicin followed by radiotherapy versus radiotherapy alone in the treatment of patients with locoregionally advanced nasopharyngeal carcinoma. Cancer. 1996; 83: 2270-83.

26. Ma J, Mai HQ, Hong MH, et al. Results of a prospective randomized trial comparing neoadjuvant chemotherapy plus radiotherapy with radiotherapy alone in patients with locoregionally advanced nasopharyngeal carcinoma. J Clin Oncol. 2001; 19: 1350-7.

27. Bossi P, Orlandi E, Bergamini C, et al. Docetaxel, cisplatin, and 5-fluorouracil-based induction chemotherapy followed by intensity modulated radiotherapy concurrent with cisplatin in locally advanced EBV-related nasopharyngeal cancer. Ann Oncol. 2011. [Epub ahead of print].

28. Bae WK, Hwang JE, Shim JH, et al. Phase II study of docetaxel, cisplatin and 5-FU induction chemotherapy followed by chemoradiotherapy in locoregionally advanced nasopharyngeal cancer. Cancer Chemother Pharmacol. 2010; 65: 589-95.

29. Hui EP, Ma BB, Leung SF, et al. Randomized phase II trial of concurrent cisplatinradiotherapy with or without neoadjuvant docetaxel and cisplatin in advanced nasopharyngeal carcinoma. J Clin Oncol. 2009; 27: 242-9.

30. Fountzilas G, Ciuleanu E, Bobos M, et al. Induction chemotherapy followed by concomitant radiotherapy and weekly cisplatin versus the same concomitant chemoradiotherapy in patients with nasopharyngeal carcinoma: a randomized phase II study conducted by the Hellenic Cooperative Oncology Group (HeCOG) with biomarker evaluation. Ann Oncol. 2012; 23: 427-35.

31. Tan T, Lim WT, Fong KW, et al. Concurrent Chemo-Radiation with or without induction gemcitabine, carboplatin, and paclitaxel: a randomized, phase $2 / 3$ trial in locally advanced nasopharyngeal carcinoma. Int J Radiat Oncol Biol Phys. 2015; 91: 952-60.

32. Usman S. Induction gemcitabine cisplatin followed by chemoradiation in locally advanced nasopharyngeal cancer, (abstract 6027). ASCO Proceedings June. 2017; $7 \mathrm{~s}$.

33. Sun Y, Li WF, Chen NY, et al. Induction Chemotherapy plus concurrent chemoradiotherapy versus concurrent chemoradiotherapy alone in locoregionally advanced nasopharyngeal carcinoma: a phase 3 , multicenter, randomized controlled trial. Lancet Oncol. 2016; 17: 1509-20.

34. Bourhis J, Daoud J, Guigay J, et al. A randomized trial of induction docetaxelcisplatin-5FU followed by concomitant cisplatin-RT versus concomitant cisplatin-RT in nasopharyngeal.

35. Mott FE, Ferrarotto R, Nguyen T, et al. Nasopharyngeal Carcinoma outcome with induction chemotherapy followed by concurrent chemoradiotherapy. Oral Oncology. 2018; 81: 75-80. 